\title{
Revue de droit de l'Université de Sherbrooke
}

\section{CRISES SANITAIRES ET RESPONSABILITÉ ÉTATIQUE ENVERS LA COLLECTIVITÉ}

\section{Lara Khoury}

Volume 46, numéro 2, 2016

URI : https://id.erudit.org/iderudit/1046331ar

DOI : https://doi.org/10.7202/1046331ar

Aller au sommaire du numéro

Éditeur(s)

Revue de Droit de l’Université de Sherbrooke

ISSN

0317-9656 (imprimé)

2561-7087 (numérique)

Découvrir la revue

Citer cet article

Khoury, L. (2016). CRISES SANITAIRES ET RESPONSABILITÉ ÉTATIQUE ENVERS LA COLLECTIVITÉ. Revue de droit de l'Université de Sherbrooke, 46(2), 261-289. https://doi.org/10.7202/1046331ar
Résumé de l'article

La crise ontarienne du SRAS a mis à l'avant-plan la responsabilité juridique de l'État dans la gestion des crises de santé publique à la lumière de ses multiples autres priorités sanitaires. Pourtant, dans le cadre d'actions collectives fondées sur la responsabilité civile intentées par des patients et professionnels de la santé ayant souffert des conséquences de cette crise, les tribunaux font preuve de déférence quant à la hiérarchie des priorités établies, à tort ou à raison, par l'État. Ce constat n'étonne pas. Les tribunaux de common law hésitent à reconnaître un devoir de diligence de la part de l'État envers des groupes de citoyens particuliers. Ils veulent ainsi prioriser la poursuite de l'intérêt collectif par les pouvoirs publics et la protection de l'autonomie exécutive et parlementaire dans la gestion de priorités conflictuelles en santé. Des considérations similaires influencent les tribunaux québécois lorsqu'ils envisagent la sauvegarde de cette autonomie par le biais de l'immunité protégeant partiellement l'État. Le présent texte vise à démontrer le faible taux de succès de l'action judiciaire collective contre l'État en santé publique, et à réfléchir à la lumière de ce constat aux doutes qu'il engendre quant au rôle que joue l'imputabilité juridique de l'État dans une gestion efficace des crises sanitaires.
Tous droits réservés @ Revue de Droit de l’Université de Sherbrooke, 2018
Ce document est protégé par la loi sur le droit d'auteur. L'utilisation des services d'Érudit (y compris la reproduction) est assujettie à sa politique d'utilisation que vous pouvez consulter en ligne.

https://apropos.erudit.org/fr/usagers/politique-dutilisation/ 


\title{
CRISES SANITAIRES ET RESPONSABILITÉ ÉTATIQUE ENVERS LA COLLECTIVITÉ
}

\author{
par Lara KHOURY*
}

La crise ontarienne du SRAS a mis à l'avant-plan la responsabilité juridique de l'État dans la gestion des crises de santé publique à la lumière de ses multiples autres priorités sanitaires. Pourtant, dans le cadre d'actions collectives fondées sur la responsabilité civile intentées par des patients et professionnels de la santé ayant souffert des conséquences de cette crise, les tribunaux font preuve de déférence quant à la hiérarchie des priorités établies, à tort ou à raison, par l'État. Ce constat n'étonne pas. Les tribunaux de common law hésitent à reconnaître un devoir de diligence de la part de l'État envers des groupes de citoyens particuliers. Ils veulent ainsi prioriser la poursuite de l'intérêt collectif par les pouvoirs publics et la protection de l'autonomie exécutive et parlementaire dans la gestion de priorités conflictuelles en santé. Des considérations similaires influencent les tribunaux québécois lorsqu'ils envisagent la sauvegarde de cette autonomie par le biais de l'immunité protégeant partiellement l'État. Le présent texte vise à démontrer le faible taux de succès de l'action judiciaire collective contre l'État en santé publique, et à réfléchir à la lumière de ce constat aux doutes qu'il engendre quant au rôle que joue l'imputabilité juridique de l'État dans une gestion efficace des crises sanitaires.

The SARS crisis in Ontario has brought to the forefront the legal responsibility of the state in the management of public health crises, in light of its many other health priorities. Yet, in the context of civil liability class actions undertaken by patients and health care professionals who have suffered the consequences of this event, courts have shown deference to the hierarchy of priorities set, rightly or wrongly, by the state. This observation is not surprising. Common law courts are reluctant to recognize a duty of care on the part of the state towards particular groups of citizens. They want to prioritize the pursuit of the collective interest by public authorities and the protection of executive and parliamentary autonomy in the management of conflicting priorities in health. Similar considerations influence Quebec courts when they seek to safeguard this autonomy by means of an immunity that partially protects the state against liability. The purpose of this paper is to point out the low success rate of class actions against the state relating to public health and to reflect, in light of this finding, on the doubts it raised regarding the role that the accountability of the state plays in the effective management of health crises.

* $\quad$ Professeure agrégée, codirectrice du Groupe de recherche en santé et droit, Faculté de droit, Université McGill, et membre associée de l'Institute for Health and Social Policy. Ce texte est inspiré d'une présentation faite dans le cadre du Colloque Les pandémies et le droit, tenu à la Faculté de droit de l'Université de Sherbrooke, le 6 novembre 2015. La recherche est à jour au 15 mars 2016. 


\section{SOMMAIRE}

\section{INTRODUCTION :}

L'ÉTAT PROTECTEUR DE LA SANTÉ

\section{PORTRAIT QUANTITATIF DES ACTIONS COLLECTIVES} EN SANTÉ PUBLIQUE CONTRE L'ÉTAT

II. EMBÛCHES RENCONTRÉES DANS LA GOUVERNANCE JUDICIAIRE DE L'ACTION ÉTATIQUE.

A. Déférence envers la hiérarchie des priorités et protection de l'autonomie exécutive

B. Priorisation de l'intérêt collectif

C. Parallèles avec les actions collectives contre les établissements hospitaliers 281 


\section{INTRODUCTION : L'ÉTAT PROTECTEUR DE LA SANTÉ}

La crise ontarienne du SRAS en 2002 et 2003 a mis à l'avantplan la question de la responsabilité juridique de l'État dans la gestion des crises de santé publique, en particulier lors de la propagation de maladies infectieuses. Elle a également permis de se questionner sur l'effet de l'imputabilité judiciaire de droit privé dans des situations où l'État doit, certes, réagir à une crise sanitaire, mais aussi gérer de multiples autres priorités de santé. Dans le cadre d'actions collectives fondées sur la responsabilité civile intentées par des patients et professionnels de la santé ayant souffert des conséquences de la crise du SRAS, les tribunaux de common law canadienne font preuve de déférence quant à la hiérarchie des priorités établies, à tort ou à raison, par l'État. Les tribunaux québécois se penchant sur la responsabilité étatique dans le domaine de la santé, pour leur part, se retirent derrière le rideau de l'immunité dont bénéficient les pouvoirs publics lorsqu'ils exercent leurs pouvoirs politiques. Bien que pour des raisons différentes, une mise en retrait judiciaire similaire s'observe dans certaines actions collectives intentées en common law contre les établissements de santé dans des contextes d'éclosion de maladies infectieuses. Le présent texte soulève la question de la responsabilité de l'État envers la collectivité en cas de crise sanitaire et plus particulièrement lorsque ces crises sont causées par la propagation de maladies infectieuses. Parce que cette question précise n'a été que très peu abordée en jurisprudence, nous entreprenons une étude jurisprudentielle plus englobante qui démontre le faible taux de succès de l'action judiciaire collective en santé publique contre l'État. Ce constat ouvre la porte à une 
discussion du rôle que peut jouer l'imputabilité juridique de l'État dans l'exhortation à une gestion efficace des crises sanitaires.

La protection de la santé publique étant d'abord, dans son entendement classique, affaire de droit public, l'État y joue un rôle prédominant. La liste des responsabilités statutaires de l'État en matière de prévention et de contrôle des infections est d'ailleurs longue et les gouvernements fédéral et provinciaux endossent une variété de rôles et de devoirs en la matière. La formulation de ces rôles et devoirs dans la législation a une importante incidence sur la responsabilité de l'État, comme on le verra. À titre d'exemple, les responsabilités législatives de l'État québécois en matière infectieuse incluent la collecte de renseignements au sujet des maladies à déclaration obligatoire, la surveillance et la vigie sanitaire, la tenue d'enquêtes socio sanitaires, et le signalement des menaces ${ }^{1}$. La province est aussi responsable des interventions en cas de menace à la santé de la population ${ }^{2}$, de la prise de mesures pour le maintien et l'amélioration de la santé (prévention, promotion, et protection) et du traitement et de la prophylaxie, incluant l'isolement obligatoire ${ }^{3}$. Elle se charge en outre des déclarations d'urgence sanitaire et des mesures à prendre dans de tels cas, des enquêtes épidémiologiques, de l'établissement et de la mise en œuvre de plans d'intervention pour le contrôle de vecteurs tels que dans le cas du virus du Nil occidental ${ }^{4}$. Enfin, elle gère le fonds québécois d'indemnisation des victimes de la vaccination ${ }^{5}$.

À l'échelle fédérale, les responsabilités statutaires incluent, par le biais de l'Agence de la santé publique du Canada (ASPC) ${ }^{6}:$ la

1. Règlement d'application de la Loi sur la santé publique, RLRQ, c. S-2.2, r. 1; Loi sur la santé publique, RLRQ, c. S-2.2.

2. Une menace à la santé de la population signifie la " présence au sein de celle-ci d'un agent biologique, chimique ou physique susceptible de causer une épidémie si la présence de cet agent n'est pas contrôlée " : Loi sur la santé publique, id., art. 2.

3. Id.

4. Id.

5. Id., art. 70-78.

6. Loi sur l’Agence de la santé publique du Canada, L.C. 2006, c. 5. 
protection et la promotion de la santé7, l'évaluation et la surveillance de l'état de santé de la population, la prévention des maladies et la préparation à l'intervention en cas d'urgence sanitaire ${ }^{8}$. Toujours par l'entremise de l'ASPC, l'État fédéral se charge d'encourager la collaboration dans le domaine de la santé publique et de coordonner les politiques et les programmes de l'administration publique fédérale en matière de santé publique ${ }^{9}$; de favoriser dans le domaine de la santé publique la consultation et la coopération avec les gouvernements provinciaux et territoriaux; et d'encourager la coopération avec les gouvernements étrangers, les organisations internationales et les autres organismes et personnes intéressés ${ }^{10}$. Enfin, le ministre fédéral de la Santé est responsable de la mise en quarantaine pour contrer les maladies épidémiques ${ }^{11}$.

L'État a donc de multiples devoirs en matière de protection de la santé des populations, incluant la prévention, la gestion et le contrôle des maladies infectieuses. Ce sont les échecs dans ce dernier domaine, et la responsabilité y afférente, qui font l'objet du présent texte. Cependant, l'analyse entreprend également des incursions dans les litiges en responsabilité contre l'État dans le domaine de la santé publique en général. Le traitement de la responsabilité étatique découlant de l'exercice des pouvoirs publics en santé permet de saisir l'ampleur actuelle des efforts vains de recherche d'imputabilité judiciaire pour la fonction exécutive. Les reproches adressés à l'État dans cette jurisprudence incluent son inaction ou son omission de règlementer ${ }^{12}$, et son défaut de mettre

7. Voir aussi la Loi sur le ministère de la Santé, L.C. 1996, c. 8, art. 4(1).

8. Loi sur l'Agence de la santé publique du Canada, préc., note 6, préambule.

9. Id.

10. Id.

11. Loi sur la mise en quarantaine, L.C. 2005, c. 20. Nous omettons dans cette énumération les responsabilités fédérales en vertu de la Loi sur les aliments et drogues, L.R.C. 1985, c. F-27, qui contient des dispositions très spécifiques dont la pertinence est plus périphérique.

12. Par exemple, dans Tonnelier c. Québec (Procureur général), 2012 QCCA 1654, on invoquait l'omission d'adopter des mesures de contrôle de qualité pour le test de dépistage du cancer du sein alors que les méthodes utilisées étaient, à la connaissance de la province, inadéquates. 
en œuvre la règlementation ou sa négligence dans cette mise en œuvre. Les retards ou la négligence dans la prise en charge de problématiques de santé sont également mis en cause ${ }^{13}$. Enfin, des recours se plaignent des décisions préjudiciables de l'État en matière de financement et d'allocation des ressources ${ }^{14}$.

Parce qu'elle montre les difficultés à entreprendre une action judiciaire collective contre l'État, l'analyse entreprise mène nécessairement à un questionnement quant au rôle que joue l'imputabilité juridique de l'État dans la gestion efficace des crises sanitaires. Ironiquement, on verra que les raisons principales invoquées pour protéger l'État contre une telle imputabilité trouvent leur source dans des facteurs favorables, en principe, à l'atteinte d'objectifs de protection de la santé. La jurisprudence reconnaît d'abord la compétence particulière des pouvoirs exécutifs en matière de gestion de la santé, par rapport à celle du judiciaire. Dans un deuxième temps, elle exprime le désir de ne pas freiner l'exercice de cette expertise et insiste sur l'importance de l'indépendance constitutionnelle dont bénéficie le décideur public à titre de représentant du citoyen dont la santé est en jeu. Enfin, les décisions judiciaires font appel à la centralité de l'intérêt collectif, composé d'un prisme d'intérêts divers et divergents, qui pourrait se trouver menacé par la reconnaissance de devoirs privés. Quant aux autres embûches, elles ne sont pas typiques à la responsabilité étatique et sans lien direct avec les objectifs de protection de la santé. Elles ont plutôt trait aux hésitations de la common law envers le préjudice moral et le "risque " de préjudice, et à des

13. Par exemple, Cilinger c. Québec (Procureur général), [2004] R.J.Q. 2943 (C.A.) (requête pour autorisation de pourvoi à la Cour suprême rejetée : (C.S. Can., 2005-07-14) 30703) concernait des temps d'attentes jugés trop longs entre les chirurgies de patientes atteintes de cancer du sein et des traitements par radiothérapie. Ces retards étaient causés, alléguait-on, par la politique de déficit zéro imposée par le gouvernement aux établissements de santé.

14. Id. 
problématiques procédurales associées à l'action collective, par ailleurs si importante en cas de dommage à large échelle.

Puisqu'il traite d'imputabilité juridique, le texte porte essentiellement sur l'étude des décisions judiciaires. De plus, ce texte étant focalisé sur la responsabilité de droit privé de l'État, il aura tendance à aborder les réponses des pouvoirs publics aux épidémies, voire aux simples éclosions, plutôt qu'aux catastrophes pandémiques. Toutefois, dans la mesure où une pandémie crée des problèmes internes à un territoire, la discussion est assurément pertinente. Ce texte démontrera le peu d'imputabilité qu'a l'État dans sa gestion des crises sanitaires, du moins par le biais des règles de droit privé. Pour ce faire, il brosse d'abord un portrait quantitatif des actions collectives entreprises contre l'État en matière de santé publique au Québec et dans le reste du Canada (I), puis se penche sur les nombreuses embûches rencontrées dans la gouvernance judiciaire de l'action étatique (II), pour conclure avec certaines questions et observations. Nous nous intéresserons particulièrement aux actions collectives intentées contre l'État en matière de gestion des maladies infectieuses afin d'englober à la fois les situations d'épidémie et de pandémie, ainsi que la dimension collective des problématiques de santé publique envisagées. Certaines poursuites individuelles d'un intérêt particulier seront toutefois prises en compte.

\section{PORTRAIT QUANTITATIF DES ACTIONS COLLECTIVES EN SANTÉ PUBLIQUE CONTRE L'ÉTAT 15}

L'État est ici envisagé au sens large comme comprenant l'ensemble des entités publiques chargées de la gestion des crises

15. Ce texte utilise le terme " action collective " plutôt que " recours collectif " afin de donner acte au changement de vocabulaire adopté par le nouveau Code de procédure civile québécois entré en vigueur le 1er janvier 2016 : Loi instituant le nouveau Code de procédure civile, L.Q. 2014, c. 1. Notons toutefois que les décisions de common law rapportées dans ce texte, ainsi que toute décision québécoise rendue avant le $1^{\mathrm{er}}$ janvier 2016, portent sur des " recours collectifs". 
sanitaires. Par exemple, au Québec, l'État dans ce contexte inclut le ministre et le ministère de la Santé et des Services sociaux, le directeur national de santé publique ${ }^{16}$, ainsi que les directeurs de santé publique ${ }^{17}$. Un portrait quantitatif des actions collectives entreprises contre l'État pour sa gestion de crises de santé publique montre que ces recours, bien qu'en augmentation, restent en nombre limité sans aucun doute en raison des embûches dont nous ferons état. Pour le Québec, une recherche dans la banque de données Soquij-Azimut - une banque de données répertoriant exhaustivement les décisions québécoises - effectuée le 21 septembre $2015^{18}$ permit de repérer treize résultats, dont trois se révélèrent pertinents en ce qu'ils portaient véritablement sur des actions collectives contre l'État dans le champ de la santé publique. Un recours concernait un cas de dépistage du cancer ${ }^{19}$, et un autre une atteinte à la santé à la suite d'un préjudice environnemental domaine pour lequel nous avons ajouté une décision non repérée ${ }^{20}$.

16. Nommé en vertu de la Loi sur le ministère de la Santé et des Services sociaux, RLRQ, c. M-19.2.

17. Nommés en vertu de la Loi sur les services de santé et les services sociaux, RLRQ, c. S-4.2, ou de la Loi sur les services de santé et les services sociaux pour les autochtones cris, RLRQ, c. S-4.1 selon la Loi sur la santé publique, préc., note 1 , art. 2.

18. Mots-clés : Recours et collectif et responsabilité (indexation) + (état ou gouvernement) et santé (résumé).

19. Tonnelier c. Québec (Procureur général), préc., note 12, rejetant l'appel de Tonnelier c. Québec (Procureure générale), 2010 QCCS 5111.

20. Coalition pour la protection de l'environnement du parc linéaire "Petit Train du Nord " c. Laurentides (Municipalité régionale de comté des), [2005] R.J.Q. 116 (C.S.); Spieser c. Canada (Procureur général), 2012 QCCS 2801 (inscription en appel : 2012-07-20 (C.A.), 200-09-007773-127). 
(2016) 46 R.D.U.S.
Crises sanitaires

et responsabilité étatique

envers la collectivité

Enfin, une dernière affaire touchait le tabagisme ${ }^{21}$. Aucun recours identifié ne mettait en cause une maladie infectieuse.

En common law canadienne, une recherche effectuée dans la banque de données Quicklaw - une banque de données répertoriant de façon exhaustive les décisions de toutes les provinces canadiennes - la même journée 22 mena à 142 résultats, dont onze concernaient des actions collectives en santé publique impliquant l'État : deux portants sur le tabagisme ${ }^{23}$; une sur une substance dangereuse (mercure) ${ }^{24}$; quatre en matière d'environnement ${ }^{25}$; une en matière de sécurité alimentaire ${ }^{26}$; et une sur la crise ontarienne du SRAS ${ }^{27}$. Enfin, deux décisions concernaient respectivement les banques de sang ${ }^{28}$ et la sécurité pharmaceutique ${ }^{29}$. Le tableau suivant résume les résultats de ces actions.

21. Canada (Procureur général) c. Imperial Tobacco Ltd., 2012 QCCA 2034, [2012] R.J.Q. 2046 (C.A.).

22. Mots-clés dans l'onglet full-text : "class /3 action \& (tort or negligence or responsibility) / 5 (state or government) $\&$ health ".

23. R. c. Imperial Tobacco Ltée, 2011 CSC 42; Knight v. Imperial Tobacco Canada Ltd., 2006 BCCA 235, 267 D.L.R. (4e) 579.

24. Holland v. British Columbia, 2009 BCCA 601, 283 B.C.A.C. 43.

25. Ring c. Canada (P.-G.), 2010 NLCA 20, 297 Nfld. \& P.E.I.R. 86; Grace c. Fort Erie (Town), [2003] O.J. No. 3475; Brooks v. Canada (Attorney General), 2010 SKCA 55, 350 Sask. R. 261, Bryson v. Canada (Attorney General), 2009 NBQB 204, 353 N.B.R. (2e) 1.

26. Los Angeles Salads Co. v. Canadian Food Inspection Agency, 2013 BCCA 34, 358 D.L.R. (4e) 581, 40 B.C.L.R. (5e) 213 (permission d'appeler à la Cour suprême du Canada refusée : [2013] S.C.C.A. No. 134).

27. Williams v. Canada (Attorney General), 2009 ONCA 378, (2009), 95 O.R. (3e) 401 (C.A. Ont.). Voir également : Abarquez v. Ontario, 2009 ONCA 374, 310 D.L.R. (4e) 726, 95 O.R. (3e) 414 (réclamation par 53 infirmières et leur famille, mais pas sous la forme d'une action collective); Laroza Estate c. Ontario, 2009 ONCA 373, (2009), 310 D.L.R. (4e) 743, 95 O.R. (3e) 764 (réclamation individuelle par une infirmière et son fils).

28. Adrian v. Canada (Minister of Health), 2007 ABQB 376, 418 A.R. 210.

29. Wuttunee v. Merck Frosst Canada Ltd., 2009 SKCA 43, 324 Sask. R. 210. Les deux recherches excluent les affaires mettant en cause un traitement médical ou de l'équipement médical, tels les implants mammaires. Voir par exemple: Attis v. Canada (Minister of Health), 2008 ONCA 660, 93 O.R. (3e) 35. 


\section{TABLEAU - ACTIONS COLLECTIVES CONTRE L'ÉTAT EN MATIĖRE DE SANTÉ PUBLIQUE}

\begin{tabular}{|c|c|c|c|c|}
\hline & Référence abrégée & $\begin{array}{l}\text { Nature de } \\
\text { l'action }\end{array}$ & Résultat ${ }^{30}$ & Sujet \\
\hline \multirow{4}{*}{ 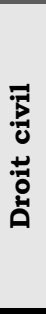 } & $\begin{array}{l}\text { Tonnelier c. } \\
\text { Québec (P. g.) }\end{array}$ & Autorisation & Rejetée & $\begin{array}{l}\text { Dépistage } \\
\text { Cancer }\end{array}$ \\
\hline & $\begin{array}{l}\text { P'tit Train du Nord } \\
\text { c. Laurentides }\end{array}$ & $\begin{array}{l}\text { Jugement au } \\
\text { fond }\end{array}$ & Accueilli & \multirow[t]{2}{*}{ Environnement } \\
\hline & $\begin{array}{l}\text { Spieser c. } \\
\text { Canada (P. g.) }\end{array}$ & $\begin{array}{l}\text { Jugement au } \\
\text { fond }\end{array}$ & Rejetée & \\
\hline & $\begin{array}{l}\text { Canada (P. g.) c. } \\
\text { Imperial Tobacco Ltd }\end{array}$ & $\begin{array}{l}\text { Recours en } \\
\text { garantie }\end{array}$ & Rejeté & Tabagisme \\
\hline \multirow{11}{*}{ 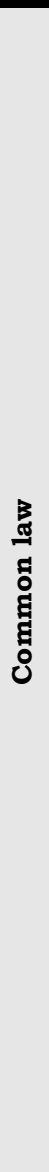 } & R. v. Imperial Tobacco & $\begin{array}{l}\text { Radiation des } \\
\text { avis de mise } \\
\text { en cause du } \\
\text { gouvernement } \\
\text { fédéral }\end{array}$ & Accueilli & \multirow[t]{2}{*}{ Tabagisme } \\
\hline & $\begin{array}{l}\text { Knight v. Imperial } \\
\text { Tobacco }\end{array}$ & Certification & $\begin{array}{l}\text { Accordée en } \\
\text { partie }\end{array}$ & \\
\hline & $\begin{array}{l}\text { Holland v. British } \\
\text { Columbia }\end{array}$ & $\begin{array}{l}\text { Jugement au } \\
\text { fond }\end{array}$ & Rejetée & $\begin{array}{l}\text { Substance } \\
\text { dangereuse }\end{array}$ \\
\hline & Ring v. Canada & Certification & Rejetée & \multirow{4}{*}{ Environnement } \\
\hline & $\begin{array}{l}\text { Grace v. Fort Erie } \\
\text { (Town) }\end{array}$ & $\begin{array}{l}\text { Motion for } \\
\text { summary } \\
\text { judgment / } \\
\text { Certification }\end{array}$ & $\begin{array}{l}\text { Certification } \\
\text { refusée }\end{array}$ & \\
\hline & $\begin{array}{l}\text { Brooks v. Canada } \\
\text { (A.G.) }\end{array}$ & Certification & Refusée & \\
\hline & $\begin{array}{l}\text { Bryson v. Canada } \\
\text { (A.G.) }\end{array}$ & Certification & Refusée & \\
\hline & $\begin{array}{l}\text { L.A. Salads Co. v. } \\
\text { CFIA }\end{array}$ & $\begin{array}{l}\text { Motion to } \\
\text { strike }\end{array}$ & Accueillie & $\begin{array}{l}\text { Sécurité } \\
\text { alimentaire }\end{array}$ \\
\hline & Williams v. Canada & $\begin{array}{l}\text { Requête en } \\
\text { rejet d'action }\end{array}$ & Accueilli & SRAS \\
\hline & $\begin{array}{l}\text { Adrian v. Canada } \\
\text { (Minister of Health) }\end{array}$ & $\begin{array}{l}\text { Certification } \\
\text { (pour } \\
\text { approbation } \\
\text { d'une } \\
\text { transaction) }\end{array}$ & Accordée & Banque de sang \\
\hline & $\begin{array}{l}\text { Wuttunee v. Merck } \\
\text { Frosst Canada Ltd. }\end{array}$ & Certification & $\begin{array}{l}\text { Refusée (en } \\
\text { appel) }\end{array}$ & $\begin{array}{l}\text { Sécurité } \\
\text { pharmaceutique }\end{array}$ \\
\hline
\end{tabular}




\section{EMBÛCHES RENCONTRÉES DANS LA GOUVERNANCE JUDICIAIRE DE L'ACTION ÉTATIQUE}

Le tableau ci-dessus laisse entrevoir que les victimes de crises de santé publique rencontrent plusieurs difficultés lorsqu'elles utilisent le droit privé comme moyen de tenir l'État imputable de ses décisions, actions et omissions. La lecture des jugements portant spécifiquement sur les maladies infectieuses permet de constater que ces embûches prennent trois visages différents. Elles ont d'abord trait à la déférence judiciaire exercée envers la hiérarchie des priorités établies par l'État et à un désir de protéger l'autonomie exécutive. De plus, elles découlent d'une volonté de prioriser la relation que l'État a avec le "public en général " et d'éliminer les interférences liées à des intérêts privés particuliers. Enfin, elles concernent l'appréciation du préjudice et de la causalité. Les défis constatés ne sont pas propres à l'action collective en ce sens qu'ils n'ont rien à voir avec le mécanisme procédural en tant que tel, à l'exception de ceux ayant trait à la causalité.

\section{A. Déférence envers la hiérarchie des priorités et protection de l'autonomie exécutive}

La première embûche a trait à la déférence dont les juges font preuve à l'égard de la hiérarchie des priorités établie par l'État et à la protection qu'ils offrent à l'autonomie exécutive. Cette déférence et protection se concrétisent par le biais de l'immunité partielle bien connue qui protège contre la responsabilité civile les décisions politiques prises par l'État. Cette immunité est bel et bien partielle, ce qui oblige à faire une difficile distinction entre la sphère politique - protégée par l'immunité ${ }^{31}$ — et la sphère opérationnelle

30. La colonne "Résultat " indique uniquement le résultat de la procédure contre l'entité étatique.

31. Des exceptions existent, en cas de mauvaise foi, de négligence grossière et d’insouciance grave: Tonnelier c. Québec (Procureur général), préc., note 12, par. 89. 
- soumise aux règles ordinaires de responsabilité civile — de l'action étatique.

Selon la Cour suprême du Canada, les décisions entrant dans la sphère politique comportent des facteurs sociaux, politiques et économiques et sont habituellement dictées par des considérations ou des contraintes d'ordre financier, économique, social et politique ${ }^{32}$. La sphère opérationnelle inclut quant à elle la mise en œuvre pratique des décisions politiques et concerne principalement l'exécution ou l'implantation d'une politique ${ }^{33}$. Malgré ces précisions, la ligne de démarcation entre les deux sphères est notoirement difficile à tracer.

Les tribunaux considèrent que la mise en balance des intérêts conflictuels sous-tendant la prise de décision en matière politique doit être laissée à ceux qui sont redevables à l'électorat. Ils justifient ainsi l'immunité par le principe de la séparation des pouvoirs : le pouvoir judiciaire ne devrait pas s'ingérer dans le travail de l'exécutif. Dans les litiges contre l'État en santé au sens large, l'immunité partielle a permis de soustraire au regard des tribunaux les décisions portant sur l'imposition de budgets et l'allocation des ressources ${ }^{34}$, et celles concernant l'établissement de priorités dans la lutte contre certaines maladies ${ }^{35}$. L'immunité empêche également les tribunaux de réviser les décisions exécutives portant sur l'évaluation des risques préalables à l'adoption, ou non, d'une règlementation ou le choix de règlementer d'une certaine manière ${ }^{36}$. Elle ne permet toutefois pas à l'État de se soustraire à

32. Brown c. Colombie-Britannique (Ministre du transport et de la voirie), [1994] 1 S.C.R. 420, p. 441.

33. Id., p. 441, habituellement les décisions opérationnelles sont le produit d'une directive administrative, de l'opinion d'un expert ou d'un professionnel, ou de normes techniques ou de la norme générale de ce qui est raisonnable.

34. Cilinger c. Québec (Procureur general), préc., note 13.

35. Id., notamment la lutte contre le cancer.

36. Sur ce point, voir Thomas Moran, Nola M. Ries et David Castle, "A Cause of Action for Regulatory Negligence? The Regulatory Framework for Genetically Modified Crops in Canada and the Potential for Regulator Liability", (2009) 6 UOLTJ 1, p. 17, 19 et 23. 
sa responsabilité lorsqu'il contrevient à une législation qu'il a luimême adoptée 37 .

Dans le domaine de la santé publique, l'immunité a permis de protéger contre une responsabilité potentielle les décisions qui ont trait à l'établissement et implantation de programmes de santé. Ce fut le cas dans l'affaire Tonnelier c. Québec (Procureur général) ${ }^{38}$. Des femmes ayant reçu un diagnostic de cancer du sein reprochaient au ministère de la Santé et des Services sociaux du Québec (MSSS) son omission d'instaurer des contrôles de qualité efficaces pour les tests pathologiques effectués pour le dépistage du cancer du sein alors qu'il savait que les mesures en place étaient insuffisantes ${ }^{39}$. La Cour supérieure et la Cour d'appel refusèrent l'autorisation d'exercer cette action collective. Pour la Cour d'appel, les décisions et actions gouvernementales pour la mise en place d'un programme de tests pathologiques, incluant la décision de rendre obligatoire la participation à des contrôles externes de qualité, relevaient de la sphère politique ${ }^{40}$. La Cour précisa qu'alors que les établissements de santé ont la responsabilité de fournir les services diagnostiques ${ }^{41}$, le MSSS se charge des grandes orientations et des décisions de politiques - dont l'implantation ou

37. Voir par exemple Association pour l'accès à l'avortement c. Québec (Procureur général), 2006 QCCS 4694, [2006] R.J.Q. 1938 (C.S.), [2006] R.R.A. 760.

38. Tonnelier c. Québec (Procureur général), préc., note 12.

39. Id., par. 7. Cette action collective avait été intentée au nom de toutes les femmes ayant reçu au Québec un diagnostic négatif de cancer du sein pour le test du marqueur biologique HER-2 ou pour les récepteurs hormonaux. Ces femmes invoquaient le stress vécu lorsque les médias annoncèrent - à la suite d'une étude rendue publique par le président de l'Association des pathologistes du Québec - que certains des résultats des tests de pathologie les concernant étaient erronés (par. 4).

40. Id., par. 64 et 87 .

41. Id., par. 34, 73, 83 et 106 . 
la mise en place des programmes de santé -, de l'allocation des budgets et des seuils de financement ${ }^{42}$.

Par contre, si l'État commet une faute dans la mise en œuvre d'une politique déjà adoptée, il est alors soumis aux règles ordinaires de la responsabilité civile ${ }^{43}$. Par exemple, la jurisprudence portant sur la transmission de maladies infectieuses laisse entendre que la mise en œuvre d'un plan de lutte contre l'épidémie se rattache à la sphère opérationnelle sujette aux règles de responsabilité civile usuelles. Encore faut-il toutefois que cette mise en œuvre relève de la responsabilité du gouvernement, et non d'une autre entité. Bien que traitant d'une poursuite individuelle, l'affaire Eliopoulos v. Ontario procure un bon exemple de cette situation. La province de l'Ontario était poursuivie par une personne décédée d'une complication d'une infection causée par le Virus du Nil occidental (VNO), et par sa famille. Les demandeurs reprochaient à la province la mise en œuvre d'un plan que le ministère de la Santé et des Soins de longue durée avait prétendument développé pour une éclosion attendue du VNO. Ce plan avait pour objectif d'encourager et de coordonner les mesures visant à réduire le risque associé au VNO en fournissant des informations aux autorités locales et au public. Cependant, la mise en ouvre de ce plan relevait non pas du ministère, mais plutôt du

42. Id., par. 73. La Cour d'appel conclut également, comme la Cour supérieure, à l'absence de causalité puisque le stress, l'angoisse et la détresse invoqués résultaient de la médiatisation et de l'information confuse et imprécise relatée par les médias, et non de la conduite du MSSS (par. 104-105 et 107).

43. C'est le cas lorsqu'il omet de faire respecter une règlementation déjà adoptée à laquelle on a contrevenu, tel que dans Bossé c. Hydro-Québec, 2012 QCCS 2919 (épandage de produits chimiques par Hydro-Québec), renversée en appel sur ce point : Hydro-Québec c. Bossé, 2014 QCCA 323 (absence de preuve d'épandage). 
(2016) 46 R.D.U.S.

Crises sanitaires

et responsabilité étatique

envers la collectivité

public, des autorités locales et des conseils locaux de santé sur qui reposaient donc les devoirs opérationnels ${ }^{44}$.

Les décisions exécutives des pouvoirs publics sont donc protégées par une immunité dès lors qu'elles impliquent l'établissement discrétionnaire de priorités, l'allocation de ressources ou la conception de plans ou programmes d'intervention. Par contre, les décisions récentes en common law canadienne justifient leur déférence envers les décisions étatiques plus fréquemment par le désir d'éviter que la reconnaissance d'une relation particulière, protégée par le droit privé, entre le citoyen et l'État interfère avec la poursuite de l'intérêt public en imposant à l'État l'obligation de tenir compte d'intérêts particuliers propres à un citoyen ou à un groupe.

\section{B. Priorisation de l'intérêt collectif}

Les tribunaux de common law hésitent traditionnellement à reconnaître un devoir de diligence de la part de l'État envers des groupes de citoyens particuliers. Ils veulent ainsi prioriser la poursuite de l'intérêt collectif par les pouvoirs publics et la gestion sans entrave des priorités conflictuelles en santé publique.

Cette deuxième embûche constitue une tendance accentuée ces dernières années dans la jurisprudence de common law. Elle trouve sa source dans le concept de proximité. L'existence d'une relation de proximité entre le demandeur et le défendeur, ici l'État, est un élément primordial pour prouver le duty of care (devoir de diligence), condition de la responsabilité sous délit de négligence en common law. Cette exigence n'existe pas en droit civil. En matière de responsabilité de l'État, l'évaluation de cette condition oblige habituellement à étudier la législation gouvernant l'action étatique afin de déterminer envers qui l'État a un devoir statutaire. Bien que la nature de ce devoir puisse varier selon la législation concernée, on observe une constante dans le domaine de la gestion des

44. Eliopoulos v. Ontario (Minister of Health \& Long Term Care) (2006), 82 O.R. (3e) 321, 43 C.C.L.T. (3e) 163 (C.A.), par. 29-30. 
maladies infectieuses, et même plus globalement en santé publique : le devoir établi par la législation habilitante est presque systématiquement envers le public en général. Les tribunaux considèrent que son devoir à l'égard du public oblige l'État à tenir compte d'une myriade d'intérêts divers et contradictoires afin de prendre la décision qui sert le mieux l'intérêt public. Par conséquent, l'imposition d'un devoir privé en faveur d'un citoyen ou d'un groupe particulier pourrait biaiser les cartes en quelque sorte, c'est-à-dire entrer en conflit direct avec ce devoir envers l'intérêt public. Deux affaires de l'Ontario et une de la Colombie-Britannique fournissent des exemples éloquents.

La première, Williams c. Canada ${ }^{45}$, porte sur l'action collective entreprise en Ontario au lendemain de la crise du SRAS. Des patients ayant contracté ce syndrome dans les hôpitaux ontariens y attaquaient les pouvoirs exercés par la province de l'Ontario en vertu de la Loi sur la protection et la promotion de la santé (LPPS) lorsqu'elle géra la deuxième éclosion de SRAS survenue en 2003. La représentante du groupe plaidait qu'elle était soumise à un risque spécifique, particulier à sa situation et à celle d'une classe limitée de personnes, soit les patients, les employés et les visiteurs de 1'hôpital, ainsi que les personnes en contact étroit avec ceux-ci. La Cour d'appel de l'Ontario conclut que la LPPS impose un devoir envers le public en général et non seulement à l'égard des catégories énumérées. Ce devoir envers le public obligeait l'État, par exemple, à aussi tenir compte dans ses décisions du besoin de maintenir l'accès aux services de santé pour la population malgré l'éclosion ${ }^{46}$.

Le deuxième exemple est en fait une poursuite individuelle, soit l'affaire Eliopoulos c. Ontario 47 déjà mentionnée. On se souviendra que le demandeur, décédé après avoir contracté le VNO,

45. Williams v. Canada (Attorney General), préc., note 27.

46. Voir aussi les recours individuels Laroza Estate c. Ontario, préc., note 27 et Abarquez c. Ontario, préc., note 27, par. 27-28 : l'imposition d'un devoir de diligence à l'égard de la sécurité des demandeurs créerait un conflit avec les devoirs généraux des provinces à l'égard du grand public.

47. Eliopoulos c. Ontario (Minister of Health \& Long Term Care), préc., note 44. 
et sa famille, arguaient que la province de l'Ontario aurait pu et aurait dû prévenir l'éclosion d'infections au VNO en 2002. Cette affaire mettait en cause, comme la précédente, des pouvoirs exercés en vertu de la LPPS. La Cour d'appel conclut que la Loi soumet le ministre de la Santé et des Soins de longue durée de l'Ontario au devoir public général de promouvoir, sauvegarder et protéger la santé des Ontariens et d'empêcher la propagation des maladies infectieuses. Ce devoir oblige le ministre à équilibrer des intérêts concurrents, mais ne donne pas lieu à une obligation de droit privé envers une personne en particulier. Elle accueillit donc la demande de rejet de la réclamation ${ }^{48}$.

Enfin, notre troisième exemple concerne, une fois de plus, une poursuite individuelle dans l'affaire Los Angeles Salads Co. c. Canadian Food Inspection Agency (Los Angeles Salad) ${ }^{49}$. Le recours fut entrepris contre l'Agence canadienne d'inspection des aliments (ACIA), Santé Canada et l'Agence de santé publique du Canada à la suite du retrait volontaire de carottes par un distributeur (Costco). Ce retrait eut lieu après la tenue d'une inspection de 1'ACIA en raison de la présence de la bactérie Shigella soupçonnée d'être liée à ces carottes. Évaluant la question de savoir si les inspecteurs fédéraux de l'ACIA ont un devoir de diligence envers les vendeurs de produits alimentaires, la Cour d'appel de la ColombieBritannique conclut que l'objectif clair de la loi applicable, la Loi sur l'Agence canadienne d'inspection des aliments, était de protéger la santé des Canadiens en prévenant la vente d'aliments contaminés au Canada ${ }^{50}$, et non de sauvegarder les intérêts économiques des fournisseurs ${ }^{51}$. Elle ajoute que reconnaître un devoir de droit privé au profit des vendeurs de produits alimentaires serait en contradiction avec ce but. De plus, un tel devoir mettrait les inspecteurs dans la position intenable d'avoir à équilibrer les

48. Voir aussi R. c. Imperial Tobacco Ltée, préc., note 23.

49. Los Angeles Salads Co. v. Canadian Food Inspection Agency, préc., note 26.

50. Id., par. 55.

51. Los Angeles Salads Co. v. Canadian Food Inspection Agency, 2011 BCSC 779, 24 B.C.L.R. (5e) 83, par. 103 (appel rejeté : Los Angeles Salads Co. v. Canadian Food Inspection Agency, préc., note 26). 
intérêts primordiaux du public avec les intérêts privés des vendeurs, et aurait ainsi un effet dissuasif sur la bonne exécution de leurs fonctions ${ }^{52}$.

Ceci étant dit, il est possible pour les tribunaux de trouver, malgré le langage législatif, une relation de proximité dans les faits entre l'État et la victime. Par exemple, cette proximité existerait en cas d'interaction ou de communication directe entre l'État et l'individu ou les individus qui subissent un préjudice dans la mise en ouvre d'une politique, surtout si la sécurité de l'individu est en jeu53. Elle pourrait aussi être présente si l'État fait des représentations particulières à un groupe ou un citoyen ou a pris des engagements envers eux à l'effet qu'il agirait dans leur intérêt, et que ces derniers s'y sont fiés ${ }^{54}$. Enfin, une relation suffisamment étroite et directe pourrait naître entre une personne et l'État si ce dernier a une connaissance personnelle des demandeurs ou de leurs circonstances, ou leur a fait des représentations ou a participé à un traitement qui a conduit à la blessure 55 .

La jurisprudence ne semble jamais avoir conclu à une proximité dans les faits dans les affaires portant sur la propagation de maladies infectieuses. De plus, dans Los Angeles Salads, la Cour d'appel de la Colombie-Britannique observe que le fait pour le public d'entrer en contact avec des entités soumises à la règlementation,

52. Los Angeles Salads Co. v. Canadian Food Inspection Agency, préc., note 26. par. 55.

53. Attis v. Canada (Minister of Health), préc., note 29, par. 66.

54. Sauer v. Canada (Attorney General), 2007 ONCA 454, 225 O.A.C. 143 (permission d'appeler à la Cour suprême du Canada refusée: [2007] S.C.C.A. No. 454).

55. $\quad$ Mitchell Estate v. Ontario (2004), 71 O.R. (3e) 571, 242 D.L.R. (4e) 560 (Ont. S.C.J.), par. 19. Voir aussi Taylor v. Canada (Attorney General), 2012 ONCA 479, 111 O.R. (3e) 161 : en présence de fausses représentations de l'État, combinées à l'omission de les corriger, en connaissance de l'existence d'un risque sérieux et continu auquel est soumis un groupe de consommateurs clairement identifiable et relativement petit. 
(2016) 46 R.D.U.S.
Crises sanitaires

et responsabilité étatique

envers la collectivité

par exemple dans le contexte d'une enquête de santé publique, ne suffit pas à établir une telle proximité dans les faits ${ }^{56}$.

Qu'en est-il lorsque la situation ex ante des demandeurs est différente de celle du public en général, par exemple en raison du fait qu'ils font partie d'un groupe soumis à un risque particulier d'atteinte à leur santé ? Cet argument fut soulevé dans Abarquez c. Ontario ${ }^{57}$. Il s'agissait d'une action entreprise contre la province de l'Ontario par 53 infirmières ayant contracté le SRAS en Ontario en 2003. La province argumenta que ses devoirs pendant la crise du SRAS n'étaient qu'envers le public en général 58 . En réponse, les infirmières plaidèrent que leur relation avec la province était différente de la relation de l'Ontario avec le grand public parce que la province avait émis des directives spécifiques aux hôpitaux pour la gestion du SRAS. L'obligation des infirmières de suivre ces directives les exposait donc, arguaient-elles, au risque particulier de contracter le SRAS. La Cour d'appel de l'Ontario, sans aborder directement ces arguments, les mit sommairement de côté en répliquant que la seule différence entre les infirmières et les membres ordinaires du public était le fait que leur profession les met plus à risque de contracter le SRAS, un risque qui n'a pas été créé par la province, mais découle de leur vocation ${ }^{59}$.

Les tribunaux de common law invoquent d'autres arguments pour justifier leur refus de reconnaître un devoir de diligence de la

56. Los Angeles Salads Co. v. Canadian Food Inspection Agency, préc., note 51, par. 106-107 et 111 (appel rejeté : Los Angeles Salads Co. v. Canadian Food Inspection Agency, préc., note 26), citant River Valley Poultry Farm Ltd v. Canada (Attorney General), 2009 ONCA 326. Permission d'appeler à la Cour suprême du Canada refusée : [2009] S.C.C.A. No. 259, 2009 CarswellOnt 6909, par. 59. De plus, les représentations faites par le site web de l'ACIA étaient insuffisantes pour créer une relation de proximité (par. 114-17).

57. Abarquez c. Ontario, préc., note 27, par. 24.

58. Voir l'argument similaire dans Williams v. Canada (Attorney General), préc., note 27, texte accompagnant la note de bas de page 46 .

59. Il n'est pas tout à fait clair si ceci était une tentative de démontrer la proximité dans les faits ou une réfutation plus directe de la conclusion à l'effet que les devoirs de la province étaient seulement envers le public. 
part de l'État envers certains groupes de victimes dans les affaires portant sur la gestion de la santé par les pouvoirs publics. Certains juges mentionnent leur inquiétude d'exposer le gouvernement à des recours privés virtuellement illimités, qui pourraient constituer un fardeau pour les finances publiques et refroidir les interventions du gouvernement ${ }^{60}$. Les tribunaux s'inquiètent en outre d'imposer un fardeau déraisonnable et non désirable à l'État, pouvant interférer avec sa prise de décision dans l'intérêt général61. On insiste aussi sur la nécessité de soupeser des revendications concurrentes pour des ressources limitées ${ }^{62}$. Enfin, les juges notent la possibilité que retenir la responsabilité de l'État mène à des effets indésirables sur la santé de la population en interférant avec le mandat principal de l'État d'établir des priorités de santé63.

Par le biais du concept de proximité, les tribunaux de common law parviennent à renforcer le choix législatif visant à prioriser l'intérêt général sans l'entrave de pressions privées canalisées par le droit de la responsabilité civile. Pour faire pression sur les pouvoirs publics afin qu'ils protègent l'intérêt privé ou individuel, il faut donc passer par la voie politique même lorsque cet intérêt concerne la protection de la sécurité et de l'intégrité.

60. Los Angeles Salads Co. v. Canadian Food Inspection Agency, préc., note 51, par. 124, confirmée sur ce point par la Cour d'appel : Los Angeles Salads Co. v. Canadian Food Inspection Agency, préc., note 26, par. 75, citant Alberta c. Elder Advocates of Alberta Society, 2011 CSC 24, [2011] 2 R.C.S. 261.

61. Eliopoulos v. Ontario (Minister of Health \& Long Term Care), préc., note 44, par. 32-33; Williams v. Canada (Attorney General), préc., note 27, par. 35 citant Eliopoulos.

62. Id.

63. Attis v. Canada (Minister of Health), préc., note 29, par. 75 : dans cette affaire portant sur la règlementation entourant les implants mammaires, la Cour d'appel de l'Ontario est d'opinion qu'imposer un devoir de droit privé à Santé Canada pourrait avoir un effet négatif (" chilling effect ") sur la santé publique et sur les intérêts collectifs en ce qu'elle porterait atteinte au mandat prédominant de Santé Canada qui vise à établir les priorités en matière de santé. Cela l'oblige à soupeser la nécessité de soulager certains individus de leurs souffrances avec le désir d'éviter tout risque (il s'agissait ici de considérations de politique résiduelle examinées à la deuxième étape du test de Anns qui permet de reconnaître un devoir de diligence dans des circonstances non encore envisagées par les tribunaux). 
(2016) 46 R.D.U.S.

Crises sanitaires

et responsabilité étatique

envers la collectivité

L'intérêt particulier s'y trouvera évidemment en compétition avec une myriade d'autres intérêts particuliers dont les pouvoirs publics ont la liberté de soupeser la valeur respective sans être soumis au regard du judiciaire. Bien sûr, ce retrait du droit privé se justifie par une reconnaissance intéressante et bienvenue de la diversité des intérêts en jeu dans le domaine de la protection de la santé et l'importance non discriminée qui doit leur être accordée. Mais elle prend naïvement pour acquis qu'une fois l'épée de Damoclès que constitue la révision judiciaire enlevée, ces intérêts seront évalués sans biais aucun. De plus, la dépendance étroite entre la définition du devoir de droit privé de l'État et l'énoncé législatif de ce devoir permet à l'État de définir lui-même les obligations juridiques auquel il est soumis et d'identifier - législativement ou par le biais des gestes qu'il pose sur le terrain - parmi les relations qu'il entretient lesquelles seront protégées par le droit privé.

Même si un devoir de diligence existait de la part de l'État envers les victimes de ses décisions politiques, et même si l'immunité lui était retirée, les tentatives de lui attribuer une responsabilité pour les conséquences préjudiciables de ses décisions ne seraient pas pour autant couronnées de succès tel que le laissent entrevoir les actions collectives entreprises en common law contre les autres acteurs institutionnels du système de santé.

\section{Parallèles avec les actions collectives contre les établissements hospitaliers}

Des actions collectives n'impliquant pas l'État, mais plutôt intentées contre les établissements de santé en common law, illustrent certaines embûches supplémentaires particulièrement pertinentes dans le contexte d'une épidémie ou d'une pandémie et qui peuvent également affecter les recours contre l'État. La reconnaissance d'un devoir de diligence de la part des établissements de santé est confrontée en effet à la hantise 
judiciaire envers le préjudice psychologique et à une recherche vaine de questions communes de causalité.

Une première difficulté survient lorsque les victimes invoquent comme préjudice indemnisable le risque de préjudice ou la peur de souffrir d'un préjudice. Certains jugements datant de la fin des années quatre-vingt-dix acceptent d'inclure dans des actions collectives des groupes de victimes invoquant la peur d'avoir contracté une maladie infectieuse à la suite de problèmes d'asepsie même si des tests subséquents révélèrent qu'ils n'avaient pas été infectés. Par exemple, dans Anderson c. Wilson ${ }^{64}$, une action collective avait été entreprise contre le propriétaire de cinq cliniques après qu'un inspecteur en santé publique identifia un possible lien entre une éclosion d'hépatite $B$ et des électroencéphalogrammes effectués dans ces cliniques. Deux groupes de patients avaient entrepris le recours, soit les patients infectés et les patients qui reçurent un avis d'infection possible, furent testés et se révélèrent non infectés. La Cour d'appel de l'Ontario accepta d'inclure dans le recours les patients non infectés. Elle invoqua pour ce faire, notamment, l'état incertain de la common law quant à la responsabilité découlant de l'infliction d'un choc nerveux. De plus, elle nota le fait que la réclamation se prêtait bien à un traitement réuni en raison de la similarité probable des réactions de chaque

64. Anderson v. Wilson (1999), O.R. (3e) 673 (C.A. Ont.) (permission d'appeler à la Cour suprême du Canada refusée : [1999] S.C.C.A. No. 476). 
membre du groupe aux avis, soit la crainte d'une infection grave et de l'anxiété pendant la période d'attente du résultat du test.

Anderson fut suivie dans au moins deux affaires subséquentes ${ }^{65}$. Dans Rideout c. Health Labrador Corp.66, une action collective avait été entreprise contre une clinique de gynécologie. Les demandeurs invoquaient le fait qu'entre octobre 2001 et mars 2003, des instruments médicaux utilisés dans la clinique pour traiter au moins 333 patients ne furent pas stérilisés adéquatement. La défenderesse diffusa un communiqué de presse et des lettres furent envoyées aux patients de la clinique pour les aviser de ce manquement, leur indiquant toutefois que le risque d'avoir contracté une infection était minime. Madame Rideout invoquait avoir entre autres avoir subi un choc nerveux à la lecture du communiqué de presse et une perte de consortium ("loss of physical and emotional consortium "67) pour elle et son mari. La Trial division de la Cour suprême de Terre-Neuve et Labrador appliqua Anderson et certifia le recours, qui fit l'objet d'une transaction en 200768 . La transaction inclut un engagement à faire des excuses, la nomination d'un représentant du public dans l'équipe responsable de développer une procédure de contrôle des

65. Rose v. Pettle, [2004] O.J. No. 739, 23 C.C.L.T. (3e) 21 : recours collectif contre un acupuncteur fondé sur une allégation de négligence à l'effet qu'il avait exposé ses patients à un risque sérieux en utilisant des aiguilles non stérilisées. Les patients plaidaient notamment avoir subi un choc nerveux après avoir été informés par les autorités de santé publique qu'ils avaient été exposés à ce risque. Cette affaire fit par la suite l'objet d'une transaction : Rose v. Pettle, [2006] O.J. No. 1612; Fakhri v. Alfalfa Canada Inc., 2003 BCSC 1717, [2003] B.C.J. No. 2618 (confirmé par 2004 BCCA 549) : recours suite à la vente de nourriture infectée par le virus de 1'hépatite A dont un des employés de la défenderesse était porteur. Un des groupes de ce recours collectif était composé de personnes non infectées qui avaient dû recevoir une injection à la suite d'un avis de santé et qui poursuivaient pour anxiété.

66. $\quad$ Rideout v. Health Labrador Corp., 2007 NLTD 116, 12 C.P.C. (6e) 91 (C.S. Lab. et T.-N.).

67. L'action per quod consortium amisit permettait historiquement à un époux de poursuivre pour la perte des services de sa conjointe, victime du délit : Lewis N. KLAR, Tort Law, 5e éd., Toronto, Carswell, p. 281-284.

68. Rideout v. Health Labrador Corp., 2007 NLTD 150, 2007 CarswellNfld 268, 822 A.P.R. 90, 270 Nfld. \& P.E.I.R. 90, 159 A.C.W.S. (3e) 789. 
infections, la délivrance par l'assureur du défendeur d'un séminaire éducatif sur le contrôle des infections, et un engagement selon lequel le PDG de la clinique discutera publiquement des changements effectués dans ses procédures de stérilisation.

Plus récemment par contre, la décision de Cour d'appel de l'Ontario dans Healey c. Lakeridge ${ }^{69}$ arrive à une conclusion différente. Cette action collective avait été entreprise contre un établissement de santé et des médecins à la suite d'expositions à un patient souffrant de tuberculose. La Cour conclut à l'absence de devoir de diligence à l'égard des victimes invoquant un "risque " de contracter la maladie, et refusa donc de certifier le recours à leur égard. Elle justifia cette décision par le fait que la common law ne reconnaît que rarement le devoir de ne pas causer de pertes "purement" psychologiques, c'est-à-dire non associées à un préjudice corporel ou matériel ${ }^{70}$. En première instance, la Cour avait également noté qu'imposer un tel devoir encouragerait les autorités de santé publique à réduire la portée des programmes de notification et augmenterait les dépenses en santé, ce qui aurait un effet sur tous les utilisateurs des soins de santé71.

L'exigence procédurale des provinces canadiennes de common law voulant que l'action collective doive soulever des

69. Healey v. Lakeridge Health Corp., 2011 ONCA 55, 103 O.R. (3e) 401.

70. Id., par. 18-19, 39-40, 60-66. Le recours fut donc certifié uniquement quant aux demandeurs ayant contracté la maladie.

71. Healey v. Lakeridge Health Corp., 2010 ONSC 725, par. 224. Voir aussi Bruce Estate v. Toderovich, 2010 ABQB 709, 497 A.R. 256 (appel rejeté : 2014 ABCA 44, 569 A.R. 78) : action collective entreprise contre un hôpital et une autorité régionale de santé par des patients traités dans un Central Sterilization Room ("CSR ") après que certains d'entre eux eurent contracté une infection à SARM et d'autres infections. Le recours fut certifié seulement pour les patients ayant été infectés. Toutefois, ce fut ici en raison d'une omission dans les allégations appuyant cette partie du recours. La Cour laisse toutefois entendre qu'elle ne serait pas favorable à une telle réclamation : "However, in my view it is impossible for the Plaintiffs to succeed on a claim that breach of a duty of care to prevent infection resulted in harm of any kind to the uninfected CSR patients. Such a breach might be indirectly relevant as giving rise to a duty to notify" (par. 40). 
questions communes aux membres du groupe ${ }^{72}$ donne également lieu à des difficultés dans les affaires de common law. Celles-ci concernent plus particulièrement la causalité qui, avance-t-on parfois, ne peut que s'apprécier individuellement pour chaque membre. Par exemple, dans l'affaire Anderson ${ }^{73}$ déjà mentionnée, les demandeurs infectés alléguaient un lien entre des cas d'hépatite $B$ confirmés et des électroencéphalogrammes ayant eu lieu dans les cliniques du défendeur. La Cour d'appel de l'Ontario ne trouve aucune question commune quant à la causalité. Cette conclusion s'appuyait sur la nécessité d'obtenir l'information sérologique pour chaque patient individuellement et d'évaluer pour chacun d'entre eux ses propres facteurs de risque - mode de vie, âge, transfusions, voyages récents dans des régions à risque, partenaires sexuels et infection - pour déterminer s'il était porteur de l'infection avant de se présenter à la clinique ${ }^{74}$. Notons que le recours fut tout de même certifié partiellement en raison de l'existence de questions communes quant à la faute.

Bien que 1'État ne fut pas impliqué dans ces affaires, cellesci permettent d'identifier des difficultés typiques des tentatives d'attribution de responsabilité à la suite de la soumission d'un groupe de personnes à un risque infectieux, du moins en common law canadienne. Donc, même en l'absence d'immunité ou en présence d'un devoir de diligence, l'indemnisation des victimes à large échelle de risque infectieux resterait aléatoire, du moins à l'extérieur du Québec.

\section{CONCLUSION}

La revue jurisprudentielle ci-dessus démontre l'existence de défis sérieux à poursuivre collectivement l'État pour les échecs perçus en santé publique. Ces défis découlent de l'immunité protégeant les décisions politiques prises par l'État dans la gestion des enjeux de santé publique, ainsi que du refus - en common law - de

72. Par exemple, la Loi de 1992 sur les recours collectifs, L.O. 1992, c. 6, art. 1 et 5 .

73. Anderson v. Wilson, préc., note 64.

74. Id., par. 28. 
reconnaître des devoirs de droit privé qui puissent entraver la poursuite de l'intérêt public par l'État dans ses décisions en santé. L'espoir de changements de l'approche judiciaire de la responsabilité de l'État en santé et en santé publique, particulièrement à l'égard de ces deux prises de position, serait illusoire, même si le nombre d'actions collectives contre les pouvoirs publics semble augmenter. Le traitement judiciaire de la responsabilité de l'État au Canada demeure, malgré ce phénomène, systématiquement restrictif.

Le constat du faible taux de succès de l'action judiciaire collective contre l'État en santé publique permet de soulever plusieurs questions intéressantes. La première concerne les potentielles distinctions dans l'imputabilité étatique générées par le bijuridisme canadien. L'immunité étatique s'applique avec force au Québec. En fait, dans la jurisprudence récente, cette immunité est, toute proportion gardée, invoquée plus souvent au Québec qu'en common law qui tend à faire échec aux poursuites contre l'État en faisant plutôt appel à l'absence de relation de proximité. Mais, mise à part cette immunité, toutes les autres embûches observées se manifestent uniquement dans la jurisprudence de common law. La situation québécoise se distingue donc à plusieurs égards.

D'abord, l'existence d'une relation de proximité entre l'État et les demandeurs n'est pas requise en responsabilité civile québécoise ${ }^{75}$, ce qui ferme la porte à l'interdépendance entre le devoir législatif et l'obligation juridique de droit privé. Par contre, la nécessité de laisser l'État soupeser sans entrave les nombreux intérêts en présence est en quelque sorte sous-entendue lorsque l'immunité trouve appui sur le besoin de protéger la prise de décision comportant l'évaluation de facteurs politiques, sociaux et économiques, ou l'appréciation des priorités dans la lutte contre les maladies et la répartition des ressources.

De plus, le droit québécois est en principe plus ouvert à l'indemnisation du préjudice purement psychologique (moral)

75. Code civil du Québec, RLRQ, c. CCQ-1991, art. 1457. 
pouvant théoriquement inclure la crainte d'un préjudice actuel ou futur pourvu qu'elle soit en relation directe avec la faute ou le fait générateur de responsabilité. La perte doit être actuelle et certaine $^{76}$, mais cette certitude ne nécessite qu'une probabilité 77 . Le dommage hypothétique ou éventuel, dont la réalisation reste trop improbable, ne peut être indemnisé 78 , mais nous ne croyons pas que cette règle fasse obstacle à l'indemnisation d'une crainte ou d'une anxiété réelle provoquée fautivement. Ce type de dommage est à distinguer des réclamations pour risque de préjudice qui, pour leur part, sont non admissibles en droit québécois à ce jour ${ }^{79}$.

Le deuxième questionnement porte sur le fossé entre l'imputabilité sanitaire et juridique de l'État et des institutions de santé résultant des embûches que nous avons identifiées. En mettant ainsi l'État à l'abri, on pourrait assister à un transfert de l'imputabilité juridique aux entités privées jouant un rôle dans la protection de la santé des populations qui, elles, ne bénéficient pas de cette clémence judiciaire. On constate ce phénomène en matière d'accès aux, et de financement des, soins de santé où la responsabilité tend à être assumée par les établissements de santé et le personnel, alors que l'État est protégé par l'immunité. On le

76. Berthiaume c. Réno-dépôt Inc., [1995] R.J.Q. 2796 (C.A.), p. 157-58 [Berthiaume-CA].

77. Jean-Louis Baudouin, Patrice Deslauriers et Benoît Moore, La responsabilité civile, vol. 1, 8e éd., Cowansville, Éditions Yvon Blais, 2014, par. 1-357; Daniel GARDNER, Le préjudice corporel, 3e éd., Cowansville, Éditions Yvon Blais, 2009, par. 64.

78. C.c.Q., art. 1611; J.-L. BaUdouin, P. Deslauriers et B. Moore, id., par. 1356 et 358.

79. Berthiaume c. Val Royal Lasalle Ltée, [1992] R.J.Q. 76 (C.S.), p. 172 [Berthiaume-CS] (aussi repris dans Berthiaume-CA c. Réno-dépôt Inc., préc., note 76, p. 52 : la réclamation incluait des dommages pour le risque augmenté de développer un cancer suite à une exposition à de la mousse isolante d'urée-formaldéhyde (MIUF). La Cour supérieure avait conclu que ce risque n'avait pas été prouvé et que la preuve était en fait à l'effet contraire (Berthiaume-CS, p. 172). La Cour d'appel confirma, avec les mentions ci-dessus (texte principal)). Voir aussi en matière environnementale : Dow Chemical Company v. Ring, Sr., préc., note 25 par. 58 (permission d'appeler à la Cour suprême du Canada refusée). 
voit bien dans Cilinger c. Québec (Procureur général)80 par exemple. La Cour supérieure et la Cour d'appel du Québec y refusaient d'autoriser une action collective entreprise contre le ministère de la Santé et des Services sociaux et le gouvernement en général par des femmes atteintes d'un cancer du sein qui invoquaient des temps d'attentes trop longs entre leurs chirurgies et leurs traitements par radiothérapie. Ces retards étaient causés, alléguait-on, par la politique de déficit zéro imposée par le gouvernement aux établissements de santé. L'autorisation fut cependant accordée contre les hôpitaux en cause ${ }^{81}$, et cette action se solda par une transaction ${ }^{82}$.

Enfin, on peut se demander si une imputabilité juridique accentuée de l'État permettrait une gestion plus efficace des crises sanitaires ou, en termes plus généraux, si le litige judiciaire collectif en responsabilité contre l'État a le pouvoir d'engendrer des changements sociaux positifs dans le domaine de la santé publique. En effet, même si elles ne sont pas couronnées de succès, les actions collectives en responsabilité civile motivent parfois, par leur seule introduction ou dans le cadre des transactions auxquelles elles peuvent donner lieu, les créateurs de risque à intervenir 83 . Malgré cela, l'on peut tout de même se demander si les embûches analysées dans ce texte sont réellement un problème. Les raisons qui les sous-tendent sont principalement liées à un désir de donner la priorité à la poursuite de l'intérêt public plutôt qu'à des intérêts particuliers, même s'ils concernent un groupe de personnes. Elles sont également associées à une reconnaissance que la gestion de la santé publique nécessite de tenir compte d'intérêts parfois contradictoires, et repose sur un ensemble de facteurs complexes à saveur politique, sociale et économique. L'exemple du SRAS est particulièrement éloquent à cet égard, en particulier l'affaire Williams dans laquelle la Cour d'appel de l'Ontario tient compte du

80. Cilinger c. Québec (Procureur général), préc., note 13. 13

81. Cilinger c. Centre hospitalier de Chicoutimi, [2004] R.J.Q. 3083 (appel rejeté : Cilinger c. Québec (Procureur général), préc., note 13).

82. Cilinger c. Centre hospitalier de Chicoutimi, 2009 QCCS 4445.

83. Voir par exemple Rideout v. Health Labrador Corp., préc., note 68 et discussion associée. 
difficile équilibre devant être atteint par l'État dans sa gestion d'une crise épidémique entre la protection des patients et du personnel médical et le maintien de l'accès aux soins de santé pendant la crise $^{84}$. En définitive, la question fondamentale qui se pose est donc de savoir qui du pouvoir exécutif et judiciaire est le mieux placé pour effectuer ces difficiles équilibrages dans le champ complexe que constitue la protection de la santé de la population.

84. Williams v. Canada (Attorney General), préc., note 27, par. 31. 